\title{
Challenges for the Promotion and Development of Traditional Chinese Medicine in Central and Eastern Europe Under the Belt and Road Initiative
}

\author{
Feifei Xue ${ }^{1}$, Xiaoyong $\mathrm{He}^{1}$, Wenzhi Hao ${ }^{1}$, Jiajia Qin ${ }^{1} \&$ Jiaxu Chen $^{1}$ \\ ${ }^{1}$ Formula-pattern Research Center, School of Traditional Chinese Medicine, Jinan University, Guangzhou, \\ China \\ Correspondence: Jiaxu Chen. E-mail: chenjiaxu@hotmail.com
}

Received: October 21, 2019

Accepted: November 19, 2019

Online Published: December 31, 2019

doi:10.5539/ass.v16n1p35

URL: https://doi.org/10.5539/ass.v16n1p35

This work was supported by the Department of International Cooperation and Exchanges of Ministry of Education of the People's Republic of China under Grant number 2059999.

\begin{abstract}
Along with the implementation of the Belt and Road Initiative, traditional Chinese medicine (TCM) is increasingly used and attracts more interest in Central and Eastern Europe (CEE). As an important bridge between different cultures, translation plays a major role in promoting TCM in CEE. However, there are some problems in the translation process hindering further promotion of TCM theories and culture in CEE. First of all, the English translations of TCM classics and textbooks lack universally accepted standards, and the quality of TCM text translation is low. Secondly, TCM translators lack sufficient training in TCM knowledge. Also, the translation of TCM materials lacks cultural connotation. Through analyzing the current problems of TCM translation in CEE, this study proposed three suggestions: strengthening the exchange between the government and experts, regulating the translation of TCM textbooks, and strengthening the training of TCM translators.
\end{abstract}

Keywords: Central and Eastern Europe, traditional Chinese medicine, translation

With the effective implementation of the Belt and Road Initiative, Chinese cultural products and practices have been accepted by an increasing number of countries in CEE and will further promote political, economic and cultural exchanges and cooperation. As an important part of the Chinese culture, TCM has also gained increasing interest and attention in CEE, serving as a channel for cultural exchange. However, on the whole, the development and scale of TCM in CEE is much slower and smaller than that in the United States, Canada and Australia, nor can it parallel with Western European countries such as Britain, France and the Netherlands (Yu, 2016). Therefore, it is necessary to learn from the above-mentioned countries about the promotion of TCM so as to further spread the TCM theories and culture and open up more opportunities for cooperation. In this process, as a medium for communication between different languages, translation is worth exploring and studying in depth.

\section{Current Situation and Problems of TCM Translation}

In recent years, along with the "going global" of traditional Chinese culture, especially TCM, the demand for translations of Chinese medical texts has increased (Wang, 2019). However, most of the translated materials are from Chinese to English with a few from Chinese to Portuguese and Spanish. Translation of Chinese medical texts to less spread Western languages is severely insufficient. Therefore, despite the continued development of translation of TCM after the implementation of the Belt and Road Initiative, there are still many difficulties and challenges.

First, the theoretical system of translation of Chinese medical texts and the translation standards for TCM terminology need to be further defined and developed. At present, there is a lack of widely-accepted translation theories in Chinese medicine, leading to low-level repeated research. Most are empirical research with few in-depth discussions on the theories of translation of Chinese medical materials. In addition, there is no standardization of translations. As Peng (2014) pointed out, available standard sets of terms for Chinese 
medicine are the basis and premise for ensuring the translation quality of Chinese medical texts. In spite of the national standards including Terms of Basic Theory in TCM, Classification and Codes for Diseases and Patterns in Chinese Medicine, Clinical Terminology of Traditional Chinese Medical Diagnosis and Treatment—Diseases, Clinical Terminology of Traditional Chinese Medical Diagnosis and Treatment-Patterns, and Clinic terminology of Traditional Chinese Medical Diagnosis and Treatment-Therapeutic methods, as well as the international term lists including the International Standard Chinese-English Basic Nomenclature of Chinese Medicine and WHO International Standard Terminologies on Traditional Medicine in the Western Pacific Region, which have provided a scientific basis for Chinese medical translation, there are many discrepancies between the standard terminologies. The lack of an authoritative standard set of terms will bring a series of problems for the teaching, research and academic exchanges of TCM.

Second, the translation of the classic works of Chinese medicine is insufficient. By now, a small number of the classic works have been translated into English; the Four Great Classics of TCM, in particular, have been translated by scholars from different countries. For example, the American, German and Chinese scholars have translated the Huang Di Nei Jing (Yellow Emperor's Inner Canon); the German scholar Paul Ulrich Unschuld has translated Huang Di Nei Jing Su Wen (Nature, Knowledge, Imagery in an Ancient Chinese Medical Text). Other classic works such as Shanghan Lun (Treatise on Cold Damage Diseases), Jingui Yaolüe (Essential Prescriptions from the Golden Cabinet), Ben Cao Gang Mu (Compendium of Materia Medica), Introductory Study of Huang Di Nei Jing, Nan Jing (Classic of Difficulties), Huang Di Wai Jing (Outer Canon of the Yellow Emperor) and Shennong Bencaojing (The Divine Farmer's Materia Medica Classic) also have translated versions. In addition, compilations and interpretations of the Four Great Classics of TCM Selected Readings from Huangdi Neijing, Selected Readings from Jingui Yaolue, Selected Readings from Shanghan Lun and Study on Warm Disease have been written in English and published. Nevertheless, all these works are rarely introduced to the countries along the Belt and Road, making it more difficult for the popularization of Chinese medicine (Bo, 2015).

Third, the training of translators in Chinese medicine is not sufficient. Translations of Chinese medical texts require a wide range of knowledge "beyond a linguistic proficiency in both Chinese and English" due to its complexity (Pritzker \& Hui, 2014). Not only do the translators need to be competent in both languages, but they should also have a background in clinical Chinese medicine, understanding of classical works and training in biomedicine (Pritzker \& Hui, 2014). However, due to the limitations of translation theory system, translation standards and translation of classic works, the training of translators in Chinese medicine fails to develop a scientific and unified program. From the perspective of the training scheme, many programs focus mainly on language training without arranging enough class hours for specialized English. Training in Chinese medicine is insufficient either, indicating that there is still much room for improvement for English majors in Chinese medicine. Medical textbook is another problem. At present, there is no standardization of translations in textbooks and medical works, and there are very few books on Chinese medicine in English. Due to the lack of an authoritative standard set of terms, different terms are used in different textbooks. Without a widely-accepted selection criterion, different universities and colleges choose their own textbooks, hindering the promotion of TCM and communications between TCM practitioners. In addition, the number of translators in Chinese medicine cannot meet the demand of the market as the Chinese medicine goes global. Currently only 13 higher education institutions of Chinese medicine in China have English majors, cultivating around 900 translators in Chinese medicine per year from 2014 to 2018. Besides, the lack of high-level competent translator has exacerbated this problem.

Fourth, more attention should be paid to the cultural contexts during the translation of Chinese medical texts. TCM has a unique medical theory system and special physiotherapy methods; it also contains terms related to Chinese philosophy of Taoism, Confucianism and Buddhism (Zhou, 2018). Therefore, Chinese medical translation not only involves the fields of medicine and translation, it also rests on cultural sensitivity. High-quality translations in Chinese medicine require the translators to have a thorough understanding of ancient Chinese, Chinese philosophy, translation theories and skills, TCM theory and clinical Chinese medicine. Such qualifications pose great challenges in finding a translator who can meet all the requirements. At present, the lack of understanding of cultural connotations in Chinese medical translation still cannot be effectively solved.

In general, the English translation of TCM texts cannot be ignored, including the translation of purely theoretical, technical, and even cultural knowledge issues that need further resolution. And we must pay attention that we should not only translate it into English, but also consider the different languages of Eastern European countries. It is undoubtedly a difficult but meaningful task. 


\section{Current Situation of TCM Translation in CEECs}

The development of TCM in 16 countries in CEE has demonstrated different momentums. As the first European nation to create detailed laws overseeing the vocational qualifications and requirements for practicing TCM, Hungary has set an example in the translation, promotion and practice of Chinese medicine. Poland and Romania, with the popularization of TCM, also have a strong foundation in academic exchanges and practice in this field. The high acceptance of Chinese medicine by Bulgaria, Estonia and Macedonia has led to deeper cooperation in TCM promotion and practice between academic institutions. TCM has also attracted interest in countries such as Latvia, Croatia, Serbia, Lithuania, Montenegro, Slovakia, Slovenia, Bosnia and Herzegovina and Albania. In this process, raising residents' awareness of Chinese medicine and expanding their acceptance is the basis for further cooperation.

\subsection{Hungary}

Hungary takes the lead in promoting TCM among the 16 CEECs. It is the first European nation to sign the memorandum of understanding over China's Belt and Road Initiative, and to create detailed laws overseeing the vocational qualifications and requirements for practicing TCM. Since the launch of the Belt and Road Initiative, TCM industry in Hungary has made great strides thanks to the efficacy of Chinese herbal medicine and its therapy, and more importantly, the efforts of TCM professionals. The importance attached to TCM in the local regions and acceptance of Chinese culture has been a key factor for the promotion of TCM, highlighting the importance of the support of the authorities and the local people.

The strengths of TCM in the treatment of difficult and complicated diseases have facilitated the popularization of TCM in Hungary, France, Czech Republic, Germany, and the United Kingdom. For example, due to the geographical reasons and reproductive culture, for those who suffer from infertility, in the case of finding no cure in Western medicine, the evidence of curative effects brought by TCM have earned trust among the residents in CEE.

The importance attached to TCM by the local government has also played a major role in TCM promotion. Since Hungarian Prime Minister Viktor Orban implemented the "Look East" policy, the Sino-Hungarian ties have been pushed to a new level. There is even a 10-minute news broadcast program hosted by the Chinese anchor in the evening news of the local national TV station. The popularity of the Chinese language not only boosts the educational and cultural exchanges between China and Hungary, it also helps the preparation of TCM translation professionals.

Since the 1980s, training programs on TCM have begun in Hungary and have developed into programs in different levels of education including higher education (such as the Heilongjiang University of Chinese Medicine, Hungary Branch and the Confucius Institute for Traditional Chinese Medicine at the University of Pecs), continuing education (limited to TCM-related associations) and heritage education. The operations of Heilongjiang University of Chinese Medicine in Budapest adopt the teaching contents and class hours according to the Chinese standards, compiling Hungarian TCM textbooks for bachelor, master and PhD programs.

Although the development of TCM in Hungary cannot parallel with that in the United States, Canada and Australia, the achievements in the field of Chinese medical translation in Hungary are prominent among CEECs. The translations of Chinese medical materials by Hungarian scholars constitute a strong basis for the promotion of TCM in Hungary and are very fruitful. For instance, Prof PálosIstván published Traditional Chinese Medicine in 1963, the earliest TCM work in Hungarian; Dr. Simoncsics Péter was the first one to employ laser acupuncture and published four books on acupuncture; Dr. Nyitrai Tamás initiated acupuncture training in 1988. The pioneer in the development of TCM in Hungary is Dr Eöry Ajándok, a well-known acupuncturist. He was the first to introduce Chinese professionals in TCM to Hungary, and to establish TCM Acupuncture Clinic in the country. He began to train professionals of Western Medicine in 1996 based on the WHO standard, and participated in the basic research of acupuncture science in the Hungarian Academy of Sciences. His books are regarded as a useful source of information in acupuncture in Hungary and the United States. Other scholars have also translated TCM classic works from other languages to Hungarian such as the Zhongyi Jingluo Zhenduan (TCM Diagnosis by meridian and collateral) translated by Prof Paul F. Volgyesi from French to Hungarian in 2011. In 2015, Mark Oravecz compiled the Chinese-Hungarian Dictionary in Chinese Medicine.

In addition to the efforts made by the Hungarian scholars, Chinese scholars also play an important role in the promotion of TCM in Hungary. Dr. Zhang Jin, Wu Binjiang, and Zhang Qinbin, famous Chinese acupuncturists have made fundamental contributions to the development of TCM in Hungary. Dr. Chen Zhen has lived in Hungary for decades and has further promoted the development of the Chinese medicine in Hungary through charity clinics and establishing a Chinese medicine product company. He published two works in Hungarian: 
Yaoshan yu Yaofang (Herbal cuisine and Recipe) and Zhongyao zai Zhongyi Linchuang shang de Yingyong (Application of TCM in clinical practice) in 2002 and 2012 respectively. Another example is Dr. Yu Funian, former honorary president of the Hungarian Traditional Chinese Medicine Association and a member of the council of the Allied Association of Hungarian Medical Societies (MOTESZ). He has been committed to promoting TCM culture in Hungary and led the HKOME association to organize seven international conferences on TCM with the local associations, greatly boosting the development of TCM, especially acupuncture in Hungary and Europe.

Therefore, Chinese medical translation has a good foundation in Hungary with frequent academic exchanges between Hungarian and Chinese scholars. Hungarian scholars who have in-depth research on Chinese medicine and Chinese scholars who have lived in Hungary for a long time are the main forces pushing forward the development of Chinese medical translation in Hungary. It is also worth noting that TCM development in Hungary does not always show positive signs. Taking acupuncture as an example, there were acupuncture training programs in the famous universities in the four major cities of Hungary. However, currently few universities continue operating these programs. Only Semmelweis University and the Confucius Institute in Budapest still have courses in Chinese medicine. TCM education is mainly conducted by unofficial institutions such as the Ontario College of Traditional Chinese Medicine Hungary campus and health training centers.

Since education is the basis for the continuous development of TCM translation, we should pay attention to the deficiencies in this area.

\subsection{Other CEECs with High Popularity of TCM Represented by Poland}

TCM has been widely accepted in Poland and Romania. Acupuncture, in particular, is very popular in both countries, which can be seen in clinical practice in the official institutions. The large scale of the Chinese medicine industry is very noticeable in Poland, where acupuncture has been carried out since the 1960s and great strides have been made later by the 1980s. Through long-term exploration and practice, the combination of Chinese herbal medicine and acupuncture has become an alternative treatment means in Poland. Thanks to the popularity of TCM, Chinese medicine practitioners in Poland have established two Chinese medicine associations, namely Polish Society of Traditional Chinese Medicine and Polish Acupuncture Society. The academic exchanges between Poland and China have also been promoted. For example, in July 2014, a Chinese medicine delegation from Gansu province visited Poland and reached an agreement with the Medical University of Lublin, the largest medical university in Poland in establishing a Chinese medicine center and training institution in Poland. In 2015, the University established the Huatuo Institute at the Medical University of Lublin with the Wenzhou Medical University.

Despite the relatively developed TCM training in Poland, there is no fixed textbook for Chinese medicine learning in Poland. Since few materials are written in Polish, Chinese medicine teaching in Poland relies mainly on English textbooks, which, as mentioned above, have no standard sets of terms and standardization of translations, leading to learning difficulties for Polish students and low accuracy of teaching. Ren et al. (2018) surveyed the Polish students who came to learn Chinese medicine in China and found that Polish students had a good understanding of acupuncture points, which should be attributed to the individual point number and the Pinyin (Chinese phonetic alphabet) name of the point. However, their understanding on the Chinese philosophy and cultural connotations involved in acupuncture varies widely.

Compared with other countries in CEE like Bulgaria, Estonia, Macedonia, Latvia, Croatia, Serbia, Lithuania, Montenegro, Slovakia, Slovenia, Bosnia and Herzegovina and Albania, Poland has a wider acceptance of TCM. However, even in countries where Chinese medicine is popularized like Poland, the translation of Chinese medical textbooks and classic works has not really started. Compared with Hungary, there are no local scholars in Poland and Romania who are keen on TCM, nor are there Chinese scholars who have played a leading role in promoting TCM. The promotion of TCM in these two countries depends mainly on English materials, indicating an urgent need for the Polish translation of Chinese medical texts.

In addition, we must pay attention to guide local doctors to learn about Chinese medicine, advocate local Chinese people to promote Chinese medicine culture, and use Hungary's successful experience as an example.

\section{Suggestions on TCM Translation in CEE}

With the issue of the first white paper on the development of TCM in China, TCM development has become a national policy, giving TCM the prime time to "go global". Intergovernmental cooperation and interpersonal exchanges have opened up more opportunities for the promotion and innovation of the TCM theories and culture. However, the low translation quality of Chinese medical materials has constituted a big challenge for the further 
promotion of TCM theories and culture. Therefore, under the background of the Belt and Road Initiative, improving translation is one of the pivotal tasks for further development of TCM in CEE.

\subsection{Promote Intergovernmental and Interpersonal Exchanges Between China and CEECs}

With the implementation of the Belt and Road Initiative, intergovernmental cooperation in Chinese medical education and research has become the main driving force for the promotion of TCM. For instance, the establishment of nearly 20 Chinese medicine centers such as the China-Czech Chinese Medicine Center has had positive and far-reaching impact on the spread of TCM culture. One of the important ways to promote exchanges in Chinese medicine consists in providing support to a number of influential non-governmental associations, which also constitute the platform to standardize translations of Chinese medical materials.

The first problem to be solved in official cooperation lies in the formulation of standards. Wang Guoqiang, director of the National Administration of Traditional Chinese Medicine said that the building of the external discourse system of Chinese medicine should make full use of the global platforms such as the WHO and International Organization for Standardization and actively participate in the formulation of relevant standards and norms by improving bilateral and multilateral exchange and cooperation mechanisms so as to play a better role in International traditional medicine (Xu \& Shen, 2018). It can be said that Setting standards for TCM translation is the basis for sustainable development of various kinds of non-government organizations and institutions.

\subsection{Develop Localized Chinese Medicine Teaching Materials, Works and Dictionaries}

The achievement of the Hungarian regulations of TCM is attributed to the scientific effects of TCM, and the tireless research, promotion and practice of TCM specialists. However, the mother tongue of CEECs is not English, and the English books in Chinese medicine have limitations. These issues constitute a huge obstacle to the understanding of the basic theoretical knowledge of Chinese medicine. By now, among the classic works of Chinese medicine that have been introduced to CEE, very few has been translated into the local languages, which is very unfavorable for the spread of TCM theories and culture. In addition, the translation quality and content of Chinese medicine works is poor; it is common to find inappropriate or even wrong content in some translation books (Qu, Tian, \& $\mathrm{Li}, 2015)$. In order to improve the translation quality of Chinese medical texts, it is of importance to organize experts who are proficient in both Chinese and the local language and Chinese and local TCM experts to participate in the translation process of Chinese medical materials. On the one hand, overseas editors can be invited to polish the translation works; on the other hand, it is necessary for Chinese well-known TCM experts to review and modify the manuscript to ensure the quality of the translation and the accuracy of cultural connotations.

\subsection{Strengthen the Training of TCM Translator for CEECS}

On the whole, the current training of Chinese medicine translators in China is still not mature. Most programs are aimed at cultivating English translators, resulting in the shortage of translators in Chinese medicine to less spread foreign languages (Hu, 2016). The lack of professionals with a high level of proficiency in TCM and languages of CEECs not only makes it difficult to advance the translation development, but also impedes the training of translation talents for CEECs, which will require long-term exploration, practice and joint efforts from governments, scientific research institutions, and Chinese communities. First, we should strengthen the top-level design to create favorable conditions for the cultivation of Chinese to less spread languages translators. Second, universities and colleges in China should attach more importance to the training of translators in Chinese medicine through conducting reforms to the class size, professional courses and language courses so as to prepare students for a wider range of career choices. Third, academic exchanges and cooperation between China and CEECs should be strengthened. Long-term cooperation programs should be designed and carried out between universities and scientific research institutions in China and CEE; more support should be provided to international and exchange students so as to prepare more qualified students for the promotion of TCM in CEE. The development of Chinese medical translation in CEE is of great importance to the sustainable development of TCM translation itself, and the implementation of the national cultural strategy. Not only is it necessary to cultivate translation professionals in Chinese medicine, but more importantly, to establish a complete translation system and mechanism.

\section{Conclusion}

All in all, under the general background of the "One Belt One Road" policy, the publicity of Chinese medicine culture has gained tremendous influence, but its influence still needs to be continuously expanded. Taking the development of TCM in Eastern European countries as an example, we must continue to strengthen the 
translation of TCM theories and strengthen the training of TCM translation talents. We must attach importance to the integration of traditional Chinese medicine propaganda work with the local language environment.

\section{References}

Bo, J. S. (2015). "Yidaiyilu" zhanlue buju xia zhongyi tushu chuban de xin jiyu (New Opportunities for the Publishing of Traditional Chinese Medicine Books under the Belt and Road Initiative). Communication and Copyright, 9, 37-38.

Hu, L. L. (2016). "Yidaiyilu” zhanlue shiyu xia Zhongyi jichu lilun kecheng dui e jiaoxue huayu tixi de goujian (The Construction of the Teaching Discourse System "Basic Theory of Traditional Chinese Medicine" to Russia from the Perspective of Belt and Road Initiative). Heilongjiang Higher Education Institute.

Peng, C. L. (2014). Cong wenhua shijiao tantao zhongyi shuyu yingyi biaozhunhua (Standardization of the English Translation of TCM Terminology from the Cultural Perspective). Journal of Hunan University of Chinese Medicine, 34(8), 62-65.

Pritzker, S. E., \& Hui, K. K. (2014). Introducing considerations in the translation of Chinese medicine. Journal of integrative medicine, 12(4), 394-396.

Qu, Q. Q., Tian, Y., \& Li, Y. J. (2015). Zai "yilaiyilu" beijing xia de xueshu tushu "zouchuqu"-yi zhongyiyao tushu "zouchuqu" gongzuo shili wei li (Academic Books "Going Global" under the "Belt and Road" Initiative-Taking the Practice of "Going Out" of Traditional Chinese Medicine Books as an Example). View on Publishing, 4, 69-71.

Ren, Z. Y., Wen, J., Cen, S. Y., \& Jiang, J. C. (2018). Cong laihua liuxuesheng kuitan zhongyiyao zai ouzhou guojia de fazhan-yi Deguo, Bolan wei li (Probing TCM Development in Europe from Overseas Students-Take Germany and Poland as Examples). Asia-Pacific Traditional Medicine, 14(10).

Wang, L. M. (2019). Cong Huoermusi fanyi liliun kan zhongyiyao fanyi yanjiu tixi (The System of TCM Translation Studies Based on James Holms's Theory of Translation Studies). Guiding Journal of Traditional Chinese Medicine and Pharmacy, 25(7), 124-127.

Xu, X. T., \& Shen, Y. D. (2018). Xiongyali zhongyiyao lifa dui zhongyi guojihua chuanbo de qishi (Inspirations on the Internationalization Communication of Traditional Chinese Medicine from Traditional Chinese Medicine Legislation in Hungary). Chinese Medicine and Culture, 13(1), 80-86.

Yu, F. N. (2016). Zhongdongou 16 guo zhongyiyao gaikuang yu fazhan zhanlue sikao (Thoughts on the General Situation and Development Strategy of Traditional Chinese Medicine in 16 Countries of Central and Eastern Europe). Guiding Journal of Traditional Chinese Medicine and Pharmacy, 22(23), 1-4.

Zhou, F. (2018). Zhongyi yingyi shi yi chuanbo yixue haishi chuanbo wenhua weizhu? zhongyi fanyi celue de yidian sikao (Is English translation of Chinese medicine for spreading the medicine or culture? On TCM Translation Strategy). WenJiao ZiLiao, 24(3), 43-44.

\section{Copyrights}

Copyright for this article is retained by the author(s), with first publication rights granted to the journal.

This is an open-access article distributed under the terms and conditions of the Creative Commons Attribution license (http://creativecommons.org/licenses/by/4.0/). 